\title{
Magnetic field effect as a probe of distance-dependent electron transfer in systems undergoing free diff usion
}

\author{
E. B. KRISSINEL ${ }^{1} \dagger$, A. I. BURSHTEIN ${ }^{2}$, N. N. LUKZEN ${ }^{3}$ and U. E. STEINER ${ }^{1 *}$ \\ ${ }^{1}$ Fakultät für Chemie, Universität Konstanz, D-78457 Konstanz, Germany \\ ${ }^{2}$ Department of Chemical Physics, Weizmann Institute of Science, Rehovot 76100 Israel \\ ${ }^{3}$ Tomographic Centre, Novosibirsk 630090 R ussia
}

\begin{abstract}
A theoretical analysis is presented of the problem of how distance-dependent electron transfer in photoinduced forward electron transfer followed by geminate backward electron transfer in liquid solution is reflected in the viscosity dependence of the magnetic field effect (MFE) on the efficiency of free radical formation $\left(\varphi_{\mathrm{ce}}\right)$ in such reactions. The stochastic Liouville equation formalism is employed to model the reaction behaviour of distance-distributed, triplet-born radical pairs (R Ps) undergoing free diffusion, distance- and spin-dependent backward electron transfer, coherent and incoherent spin evolution in the ps time domain. In comparison with real systems the spin situation is simplified by reducing it to a two state $\left(S, T_{0}\right)$ problem, yet it is parametrized in a way that allows sensible comparison of the results with those of recent experiments. It is predicted that the MFE on $\varphi_{\mathrm{ce}}$ exhibits characteristic minima in the MFE versus viscosity curves, and it is verified in detail that this feature is peculiar to the diffusional model with distance-dependent electron transfer, i.e. cannot be reproduced with the simpler ('exponential') RP model employing distance-independent rate constants. Thus, the MFE versus viscosity curves are established as a genuine fingerprint of distance-dependent electron transfer. The theoretical results compare favourably with recent experimental results obtained with $\mathrm{Ru}^{\mathrm{III}}$ complex/methylviologen R Ps.
\end{abstract}

\section{Introduction}

One of the most prominent features distinguishing electron transfer processes from other elementary intermolecular chemical reactions is the fact that they do not require close contact between the reacting particles because of the extraordinary capability of electrons to tunnel through sizeable distances from one binding site of the electron to another [1-3]. Typical for such tunnelling processes is an exponential decrease of the rate constant $k_{\text {et }}$ of electron transfer with the distance $r$ [4-6]. In general the decay of $k_{\text {et }}$ with distance is due to a change of the electronic factor and the nuclear factor [7]. The function may become non-exponential in case of strongly exergonic electron transfer $[8,9]$. We will not consider the latter behaviour in the present paper but confine ourselves to the exponential expression

$$
k_{\mathrm{et}}(r)=W_{0} \exp [-(r-a) / L],
$$

where $W_{0}$ is the rate constant at contact distance $a$, and $L$ is a characteristic length parameter, typically between

*Author for correspondence. e-mail: steiner@ch-lu.chemie. uni-konstanz.de

$\dagger$ Humboldt fellow, on leave from the Institute for Water and Environmental Problems, Barnaul 656009, R ussia.
$0.5-1 \AA[6,10,11]$ (it may become larger if there is strong superexchange with the intervening medium [12-15]). Experimental verifications of such exponential dependence have been provided for electron transfer reactions in systems with frozen donor-acceptor distance distribution as in solid solutions [16-18], and in systems with fixed distances as in rigid supramolecular systems [1923] (for a review see [24]) and in biological systems $[24,25]$. In liquid solution the distances between electron donors and acceptors are under continuous change as a consequence of diff usion. The solid state approximation of calculating the (time-dependent) reaction rate of an ensemble by averaging the distance-dependent rate constant over a quasi-immobile yet, as the reaction proceeds, depleting distribution, breaks down on a timescale long with respect to $a L / D$, where $D$ is the relative diffusion constant of the reactants [26-28]. The general kinetic treatment has to account for changes of the pair distribution function due to both distancedependent electron transfer and diffusion. Principally, the most detailed and best time-resolved kinetic information comes from photoinduced electron transfer reactions, where the luminescence of an excited donor or acceptor is a most sensitive kinetic probe [10, 29-32]. In general its decay exhibits an initial part with time 
dependent rate constant, which is the analogue of what happens in solid solutions and normally is denoted as 'static' quenching. After longer times, depending on the concentration of the quencher, the kinetics approach a limiting type of behaviour with a time-independent rate constant $k_{q}$. For the latter stage, which dominates the whole decay if the quencher concentration is dilute, two limiting situations ensue, known as the limits of kinetic control (fast diffusion, i.e., $\left.W_{0}(a L / D) \ll 1\right)$ and of diff usion control (fast reaction, i.e., $\left.W_{0}(a L / D) \gg 1\right)$. If the value of $W_{0}$ is not known exactly, a definite conclusion regarding contributions of 'remote' electron transfer cannot be derived from the value of $k_{\mathrm{q}}$ in the case of kinetic control. However, in the case of diffusion control, $R$ eff values obtained from the stationary value of $k_{\mathrm{q}}$ through the relation $k_{\mathrm{q}}=4 \pi R_{\text {eff }} D N_{\mathrm{A}}$ represent a conclusive indicator of remote electron transfer taking place if $R_{\text {eff }}$ is larger than the contact distance $a$ [9].

Detailed kinetic treatments of irreversible excited state quenching by distance-dependent electron transfer have been presented by a number of authors $[10,30,33-$ 36]. However, the general scenario of photoinduced electron transfer is still more complex. The primary products of such reactions are radical (ion) pairs (R Ps), for which recombination to the initial ground state reactants (or sometimes with one of the regenerated reactants being formed in a low lying excited state) by backward electron transfer usually has a high thermodynamic driving force. After photoinduced forward electron transfer, there is a competition between backward electron transfer, i.e., charge recombination, and diffusive separation to free radical ions. In order to calculate the overall yield of free radical formation (otherwise denoted cage escape efficiency or survival probability of the RP after infinite time, $\varphi_{\text {ce }}$ ) in the most general way, one has to account for remote electron transfer in the forward and in the backward electron transfer reaction. Such treatments have been provided in [29, 37-42] and have made it possible to calculate the populations of excited states $\left\langle P_{\mathrm{ex}}(t)\right\rangle$ and of radical ion pairs $\left\langle P_{\mathrm{RP}}(t)\right\rangle$. It has been demonstrated how the stationary quenching constant $k_{q}$ and the cage escape efficiency $\varphi_{\text {ce }}$ are affected by a variation of the basic parameters $a, W_{0, \mathrm{f}}, L_{\mathrm{f}}, W_{0, \mathrm{~b}}, L_{\mathrm{b}}, D$, i.e., encounter distance, parameters of the distance laws for forward and backward electron transfer, and relative diffusion coefficient.

In principle, the most complete experimental information is obtained from a direct measurement of the timedependent functions $\left\langle P_{\mathrm{ex}}(t)\right\rangle$ and $\left\langle P_{\mathrm{RP}}(t)\right\rangle$. However, specific kinetic information about the backward electron transfer process does show up only if the forward electron transfer can be made faster than the geminate recombination. This requirement necessitates very high quencher concentrations, and is impossible even for the highest quencher concentration if $W_{0, \mathrm{f}}<W_{0, \mathrm{~b}}$. In that case direct time-resolved information on the backward electron transfer process cannot be obtained because it will be always masked by the slower forward electron transfer. If $W_{0, \mathrm{f}}>W_{0, \mathrm{~b}}$, for sufficiently high quencher concentration the actual generation of the RPs is faster than their (geminate) recombination. However, high quencher concentrations may represent an undesired condition, since it means that the environment of the excited reactants and of the created R Ps is affected significantly by the presence of unreacted accessory quencher molecules which, in other words, alter the character of the medium. On the other hand, working with dilute solutions usually allows the determination of the stationary quantities $k_{\mathrm{q}}$ and $\varphi_{\mathrm{ce}}$ only. For the purposes of an experimental determination of the multiparametric details of diffusion and distance-dependent forward and backward electron transfer, $k_{\mathrm{q}}$ and $\varphi_{\mathrm{ce}}$ are somewhat global quantities from which it is difficult, in general, to infer unambiguous information on remote electron transfer [29].

Specific sources of additional experimental information on the role of diffusion in $\mathrm{R} P$ recombination can be found in the field of spin chemistry (cf. [43] and references therein). Typical spin chemical effects like chemically induced magnetic polarization of nuclei and electrons, magnetic isotope effects, and magnetic field effects on chemical reaction rates and yields of reactions involving radical pairs as intermediates [44] are based largely on the so-called radical pair mechanism, which associates the principles of spin conservation with chemical reaction, so that the R P's electron spin alignment, triplet or singlet, determines the chemical reactivity towards recombination. For example, the recombination product being usually diamagnetic means that RPs with singlet spin are reactive whereas R Ps with triplet spin are not, and triplet-singlet spin conversion, e.g., driven by hyperfine interaction, will cause a change of reactivity. Observable spin chemical effects ensue if the timescale of such spin processes matches that of the decay of spatial correlation of geminate RPs, and if the rates of the spin processes depend on nuclear spin states or can be altered by the application of external magnetic fields.

Spin-dependent reactions controlled by hyperfine interaction are very sensitive to exchange interaction with its exponential distance dependence [45-48]. Usually, in contact situations the singlet-triplet splitting caused by exchange interaction is much stronger than the hyperfine coupling energies, so that triplet-singlet processes cannot occur by that mechanism and triplet $R$ Ps remain unreactive and will diffuse apart unreacted. On the other hand, at a distance where the exchange 
interaction is sufficiently small and $T-S$ conversion can occur, the separation of the two radicals may be already too far for recombination to take place, so that the radicals have to approach each other once again in order to react $[49,50]$. From this qualitative scenario it is clear that R Ps created with unreactive multiplicity and recombining only after suitable spin conversion in some distance and successive re-encounter will be sensitive probes of specific diffusional trajectories involving separation and geminate re-encounter. To deal with the effects of distance-dependent exchange interaction, various theoretical methods and models have been applied, ranging from contact approximation-with exchange interaction infinitely high at contact and zero elsewhere-to more realistic, truly distance-dependent functions (cf. [43], table 20). Theoretical interpretations of experimental results on magnetic field dependent kinetic effects with chemically linked radical pairs have shown that such effects indeed map the (distance-distribution weighted) profile of the exchange interaction ('exchange spectrum' [51-56]) which, however, often may appear distorted due to dynamic effects of diffusional or conformational motion if the dynamics of flexible chains linking the two radicals are concerned.

By analogy with such investigation s of experimentally observable consequences of distance-dependent exchange interaction, one might infer that the spinchemically controllable time-dependent reactivity of freely diffusing radical pairs can be exploited also for probing the distance-dependence of electron transfer rates. To our knowledge this aspect has not been investigated systematically so far. Some contrasting conclusions have been reported for flexibly linked radical pairs. Whereas Bittl and Schulten [52] found no difference between distance-dependent and distance-independent electron transfer, an observation which they assigned to the effect that the stochastic motion averages the decay rates in the various conformations of the biradical to one efficient decay rate, Staerk et al. [55] found it essential to use contact reaction behaviour in order to reproduce the experimental results. This was not possible with distance independent recombination. In these two studies the timescale spans several ns. In the present paper we will deal with processes on the ps time domain. We will refer to experimental results obtained with a spin-chemically quite different and less common type of spin probe, i.e., radical pairs involving strongly spin-orbit coupled paramagnetic low-spin complexes of $\mathrm{Ru}^{\mathrm{III}}$ and $\mathrm{Fe}^{\mathrm{III}}$ [57-61]. Although being formal $s=1 / 2$ species and therefore spin-chemically fitting neatly into the radical pair concept, these d-electrontype radicals differ from purely organic radicals in two quantitative aspects: they exhibit extremely short transversal as well as longitudinal spin relaxation times of the order of a few to several tens of picoseconds [62] and they have strongly anisotropic $g$ tensors with components that may differ from the $g$ factor of organic radicals by a value of 1 or more. In R Ps involving such d electron radicals, the extreme $g$ tensor properties can be exploited for stimulating very fast magnetic field induced tripletsinglet mixing, the frequency of such spin processes being given approximately by

$$
\omega \approx \Delta g B_{0} / \bar{h}
$$

where $\beta$ is Bohr's magneton and $B_{0}$ the magnetic field. With the difference in $g$ factors, $\Delta g$, of the order of 1 it follows that in a field of $1 \mathrm{~T}, \omega_{\mathrm{TS}} \approx 10^{11} \mathrm{rad} \mathrm{s}^{-1}$ which matches the order of the natural spin relaxation rate in these complexes. Thus the spin motion, and hence the $\mathrm{R} P$ reactivity, can be controlled magnetically on the timescale of a few picoseconds, i.e., down to times where the diffusive length is of the order of a few angstroms, which matches the scale of the distance law of remote electron transfer.

As a further advantage of the R P systems with bulky metal complexes we may note that distance-dependent exchange interaction is of minor importance here. This is due to the closest approach of the two radical centres remaining quite considerable if the paramagnetic metal is surrounded by bulky ligands as in case of the $\mathrm{Ru}$ trisbipyridine complex $\left(\mathrm{Ru}(\mathrm{bpy})_{3}^{2+}\right)$. On a timescale of some $10 \mathrm{ps}$, effects of exchange interaction would be relevant only if the $J$ splitting were equivalent to $\geq 0.1 \mathrm{~T}$, which is higher than expected on the basis of exchange energy distance functions empirically calibrated with chemically linked R Ps [63-66].

In this paper we are referring specifically to experimental results regarding the viscosity dependence of the magnetic field effect on the cage escape yield of free radical ions $\left(\varphi_{\mathrm{ce}}\right)$ created in the electron transfer reaction between photoexcited $\mathrm{Ru}(\mathrm{bpy})_{3}^{2+}$ and methyl viologen $\left(\mathrm{MV}^{2+}\right)$ according to the overall equation [67]

$$
{ }^{3}\left[\mathrm{Ru}^{\mathrm{II}}(\mathrm{bpy})_{3}^{2+}\right]+\mathrm{MV}^{2+} \rightarrow \mathrm{Ru}^{\mathrm{III}}(\mathrm{bpy})_{3}^{2+}+\mathrm{MV}^{+} \text {. }
$$

The essential experimental results are reproduced in figure 1 , where in figure $1(b)$ we have adapted the representation to indicate more clearly the viscosity dependence of the magnetic field effect. In this paper we will demonstrate that the experimentally observed family of curves of magnetic field effect versus viscosity can be considered as a 'fingerprint of distance-dependent electron transfer'. It must be emphasized, though, that the purpose of the present work is not to provide a quantitative fit of the available experimental results, but to analyse in detail the principal kinetic consequences arising from a unified treatment of distance-dependent forward and backward electron transfer in conjunction with a magnetically controlled R P reactivity. In order to 


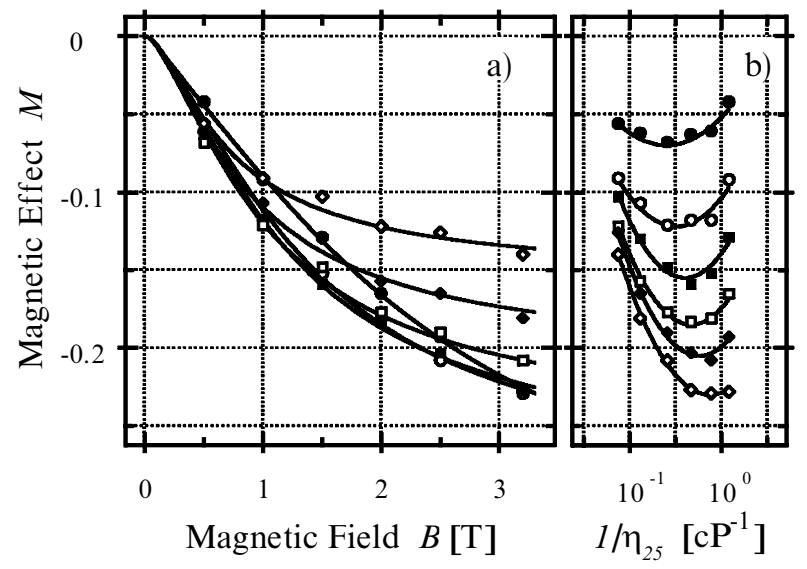

Figure 1 $(a)$. Magnetic field dependence of MFE on $\varphi_{\text {ce }}$ in reaction (3) observed for solvent mixtures $\left(\mathrm{H}_{2} \mathrm{O}: \mathrm{ACN}, 1: 1\right.$, with ethylene glycol) of different viscosities (in cP): $\diamond, 13.40 ; \diamond, 7.68 ; \square, 3.88 ; \square, 2.15 ; \bigcirc, 1.29$; - 0.83 . The solid lines represent best fits obtained on the basis of the exponential model (diagram adapted from [67]). (b) Data of part (a) represented as viscosity dependence of the MFE for various magnetic fields (in T): $\diamond$, $3.2 ; \diamond, 2.5 ; \square, 2.0 ; \square, 1.5 ; \bigcirc, 1.0 ; \bigcirc, 0.5$. The solid lines represent second-order polynomial approximations drawn to aid the viewer.

make the analysis more transparent we have resorted to a somewhat simplified, albeit still realistically parametrized, model of spin motion.

\section{Theoretical background}

In liquid solution, electron transfer between photoexcited electron donors or acceptors and ground state quenchers results in the creation of $\mathrm{R} P \mathrm{Ps}$ within a range of distances between the two radicals, normally less than a few $\AA$ wider than the van der Waals contact distance. The R P will be assumed to originate with exactly the spin multiplicity of its precursor. However, once formed, the spin state of the RP will evolve in time and the interradical distance will be stochastically modulated due to the diffusive motion of the radicals. As long as the distance between the radicals is sufficiently short there will be the possibility of backward electron transfer leading to the ground state of the reactants. The rate constant of the latter process depends on the distance as well as on the current value of the total electron spin of the RP. That fraction of the overall generated RPs which survive after long times defines the escape efficiency $\varphi_{\text {ce }}$, which is the observable quantity of interest here. Interactions between radicals having originated in different pairs, i.e., the bulk recombination kinetics of radicals, will not be considered in the present treatment.

The kinetic evolution of an ensemble of R Ps with respect to the degrees of freedom described above is conveniently described by means of a distance-dependent spin density matrix $\hat{\rho}(r, t)$ :

$$
\frac{\partial \hat{\rho}}{\partial t}=\hat{\mathscr{B}} \hat{\rho}-\mathrm{i}[\hat{H}, \hat{\rho}]_{-}-\frac{1}{2}[\hat{W}, \hat{\rho}]_{+}+\hat{\mathscr{R}} \hat{\rho}+\hat{s}
$$

where $\hat{\mathscr{B}}$ designates the stochastic operator of relative motion of the radicals, $\hat{H}$ represents the spin Hamiltonian of the RP, $\hat{W}$ is the reaction operator of BET and $\hat{\mathscr{R}}$ is the relaxation operator. Commutator and anticommutator $[x, y]_{ \pm}=x y \pm y x$ are defined as usual. The term $\hat{s}$ is a source term describing the creation of R Ps as a function of time and distance. At $t=0$ we will assume $\hat{\rho}(r, 0)=0$. The normalization of $\hat{s}$ (determining the normalization of $\hat{\rho}(r, t)$ ) will be such that

$$
\operatorname{tr}\left\{\int_{0}^{\infty} \mathrm{d} t \int_{\mathrm{a}}^{\infty} \hat{s}(r, t) \mathrm{d}^{3} r\right\}=\varphi_{\mathrm{q}},
$$

where $\varphi_{\mathrm{q}}$ corresponds to the fraction of excited states converted to R Ps by the quenching process.

In the present study we restrict ourselves to a simple model of an R P having only two electron spin levels, one reactive $(S)$ and the other unreactive $(T)$, which may be envisaged as a restriction to $S$ and $T_{0}$, as is often done with normal organic R Ps in the high field limit, when $T \pm$ are strongly decoupled from $T_{0}$ and $S$, so that coherent and incoherent transitions between them become negligibly slow. However, in the R Ps under consideration the radical moiety represented by the paramagnetic complex is susceptible to very fast, spin-orbit coupling induced spin relaxation. Thereby effective, magnetic field independent spin relaxation occurs among all spin sublevels of the RP. Because of this, $T \pm$ states should be taken into account for all fields. Although available for numerical solution (cf. [57]), the full four-level system is rather hard to analyse. In this paper, rather than fitting the experimental results obtained in [67] for the viscosity effect on the efficiency of separation precisely, our aim is a qualitative study of the general features borne out by the viscosity dependence of the magnetic field effect. We believe that a simplified two-level $\left(S, T_{0}\right)$ model with spin relaxation, coherent $S \leftrightarrow T_{0}$ conversion and $S$-selective recombination is sufficient for such a qualitative study.

In the two-state basis the spin Hamiltonian has the form

$$
\hat{H}=\frac{1}{2}\left(\begin{array}{ll}
0 & \omega \\
\omega & 0
\end{array}\right),
$$

where $\omega$ is the frequency of $S-T_{0}$ transitions. In case of the $\Delta g$ mechanism, relevant to the systems of interest here, $\omega$ is proportional to the external magnetic field (cf. equation (1)). The reaction operator $\hat{W}$ allows BET to occur only in singlet R Ps: 


$$
\hat{W}=\left(\begin{array}{cc}
k_{\text {bet }}(r) & 0 \\
0 & 0
\end{array}\right) .
$$

The relaxation operator $\hat{\mathscr{R}}$ describes equilibration among the spin states due to paramagnetic relaxation. This operator may be obtained from that of the full four-level system (cf. [57]). The decoupling of $T \pm$ sublevels is achieved by setting the longitudinal relaxation time $T_{1}$ equal to infinity and keeping only $T_{2}$ (here denoted by $T$ ). Then the nonzero elements of $\hat{\mathscr{R}}$ are:

$$
\begin{aligned}
& \hat{\mathscr{R}}_{S S, S S}=\hat{\mathscr{R}}_{T_{0} T_{0}, T_{0} T_{0}}=-\hat{\mathscr{R}}_{S S, T_{0} T_{0}}-\hat{\mathscr{R}}_{T_{0} T_{0}, S S}=-\frac{1}{2 T}, \\
& \hat{\mathscr{R}}_{S T_{0}, S T_{0}}=\hat{\mathscr{R}}_{T_{0} S, T_{0} S}=-\frac{1}{T} .
\end{aligned}
$$

In accounting for the Brownian motion of the radicals, causing a stochastic variation of the interradical distance, we will make use of the widely accepted approximation of continuous diffusion [26]. Within this approach, that will be referred to as the 'diffusional model', the Smoluchowski operator $\hat{\mathscr{B}}$ has the following form $[26,68]$ :

$$
\hat{\mathscr{B}}=\frac{1}{r^{2}} \frac{\partial}{\partial r} D r^{2}\left(\frac{\partial}{\partial r}+\frac{1}{k T} \frac{\partial V}{\partial r}\right),
$$

where $r$ is the interradical distance, $D$ the coefficient of relative diffusion of the two radicals, and $V$ the interradical potential. Substitution of equation (9) into (4) yields a partial differential equation which needs specification of two boundary conditions and the source term $\hat{s}$. The boundary conditions may be specified by taking into account that there are no RPs with infinite interparticle distance and by assuming reflecting encounters of the radicals at contact radius $a$, i.e., the disappearance of RPs is exclusively represented by the action of the reaction operator $\hat{W}$ for the BET process:

$$
\hat{\rho}(r \rightarrow \infty, t)=0, \quad \frac{\partial \hat{\rho}(a, t)}{\partial r}+\frac{\hat{\rho}(a, t)}{k T} \frac{\partial V}{\partial r}=0 .
$$

The term $\hat{s}$, describing the generation of R Ps in space and time, is derived from a distance and diffusion dependent treatment of the forward electron transfer according to the technique described in [40]. Let $n(r, t)$ denote the distribution function of pairs of excited molecules and electron transfer quenchers, normalized such that $n(\infty, t)=1$. Then, since we consider the case of photogenerated reactive triplet states, in our two-level spin system the $T_{0}$ substate of the $\mathrm{RP}$ is populated according to

$$
\hat{s}_{T_{0} T_{0}}(r, t)=k_{\mathrm{fet}}(r)_{n}(r, t)_{c} \mathrm{Q}^{N}(t),
$$

where $k_{\text {fet }}(r)$ is the distance-dependent rate constant of FET, $c_{\mathrm{Q}}$ is the bulk concentration of quenchers, and ${ }_{N}(t)$ is the population of excited donors, subject to the initial condition $N(0)=1$. The time dependence of the excited state population $N(t)$ is described by the following differential equation:

$$
\dot{N}(t)=-k_{\mathrm{I}}(t)_{c} \mathrm{Q}^{N}(t)-{ }_{N}(t) / \tau_{\mathrm{D}},
$$

where $T_{D}$ is the spontaneous decay time of the photoreactive molecules and $k_{\mathrm{I}}(t)$ the nonstationary rate constant of FET:

$$
k_{\mathrm{I}}(t)=\int_{a}^{\infty} k_{\mathrm{fet}}(r) n(r, t) \mathrm{d}^{3} r .
$$

In this study we assume exponential dependence of the forward and backward electron transfer rate constants on the interradical distance $r$ :

$$
k_{\text {fet }}(r)=k_{\text {bet }}(r)=W_{0} \exp \left(-\frac{r-a}{L}\right) .
$$

The pair distribution function $n(r, t)$ obeys the Smoluchowski-Einstein equation

$$
\frac{\partial n(r, t)}{\partial t}=\hat{\mathscr{L}}_{n}(r, t)-k_{\mathrm{fet}}(r)_{n}(r, t)
$$

with the boundary conditions

$$
{ }_{n}(r \rightarrow \infty, t)=1, \quad \frac{\partial n(a, t)}{\partial r}+\frac{n(a, t)}{k T} \frac{\partial V}{\partial r}=0,
$$

and the initial condition

$$
{ }_{n}(r, t=0)=1 \text {, }
$$

corresponding to uniform initial distribution of donoracceptor pairs at the instant of photoexcitation.

Equations (1) and (9)-(17) represent the extension of the unified theory of forward and back electron transfer, developed in [39-41], to the case of spin-dependent BET. Previously (see $[69,70]$ ) the unified theory had been extended from the spin-independent case to the case of a two-level RP with interlevel transitions described by rate constants. For R Ps of the type which is of interest here, such a rate description of singlet-triplet transition s would be reasonable only for low magnetic fields where spin relaxation is the dominating process between the levels. For relaxation times $T$ of the order of $10 \mathrm{ps}$ the condition $\omega T \ll 1$ yields an upper field limit of $0.5 \mathrm{~T}$. Since the magnetic fields of interest here are much higher (cf. figure 1) it is important that the coherent nature of the spin motion in the field is taken into account explicitly.

We will not consider the explicit time dependence of radical pair formation and recombination. Therefore we reduce equation (4) to time-independent form by integration over time, yielding:

$$
-\hat{\rho}_{0}=\hat{\mathscr{L}} \hat{\sigma}-\mathrm{i}[\hat{H}, \hat{\sigma}]_{-}-\frac{1}{2}[\hat{W}, \hat{\sigma}]_{+}+\hat{\mathscr{R}} \hat{\sigma},
$$




$$
\begin{gathered}
\hat{\sigma}(r)=\int_{0}^{\infty} \hat{\rho}(r, t) \mathrm{d} t, \\
\hat{\rho}_{0}(r)=\int_{0}^{\infty} \hat{s}(r, t) \mathrm{d} t .
\end{gathered}
$$

Integration of equations (15), (13), and (12) is necessary for obtaining the RP's initial distribution function $f_{0}(r) \equiv 4 \pi^{2} \operatorname{tr}\left(\hat{\rho}_{0}(r)\right)$. Finally, the efficiency $\varphi_{\mathrm{fr}}$ of free radical formation from each excited molecule is obtained as the difference of FET and BET efficiencies:

$$
\varphi_{\mathrm{fr}}=\varphi_{\mathrm{q}}-\varphi_{\mathrm{rec}}
$$

where

$$
\begin{aligned}
\varphi_{\text {rec }} & =\operatorname{tr}\left(\int_{0}^{\infty} \mathrm{d} t \int_{\mathrm{a}}^{\infty} \frac{1}{2}[\hat{W}(r), \hat{\rho}(r, t)]_{+} \mathrm{d}^{3} r\right) \\
& =\operatorname{tr}\left(\int_{\mathrm{a}}^{\infty} \frac{1}{2}[\hat{W}(r), \hat{\sigma}(r)]_{+} \mathrm{d}^{3} r\right) .
\end{aligned}
$$

The quantity $\varphi_{\text {fr }}$ may be factorized as

$$
\varphi_{\mathrm{fr}}=\varphi_{\mathrm{q}} \varphi_{\mathrm{ce}},
$$

so that the characteristic quantity $\varphi_{\text {ce }}$ determined in the experiments as a function of the magnetic field can be obtained by combining the results of equations $(18-22)$ and (5).

Except for some limiting cases, it is virtually not possible to solve equations (4) and (9-17) analytically. We solved them numerically employing a non-equidistant finite-difference scheme for the space coordinate $r$ and the implicit Euler scheme with variably adjusted timesteps for joint integration of equations (12) and (15). Details of the numerical procedures employed may be found elsewhere [71, 72]. For the special case of R Ps generated and recombining in contact only, Mints and Pukhov [73] gave an analytical solution. Our numerical results are in excellent agreement with the analytical solution for that special case.

In the spin chemical literature frequently it has been found convenient to resort to the simplest approximation one may choose for describing the kinetics of recombination and separation of $\mathrm{R} P \mathrm{P}$, i.e., a representation employing first-order rate constants, which is tantamount to ignoring the interradical distance as a continuous degree of internal freedom of this species. This simplified model, which distinguishes only the discrete species R Ps, free radicals and recombined states, has been termed the 'exponential model', since it leads to exponential decay of the R Ps. The applicability of the exponential model to liquid phase reactions has been discussed widely in the literature (see, e.g., [74, 75]). It works well when details of the stochastic distance variation of the $\mathrm{RP}$ within the reaction zone do not affect the probability of BET. This is the case when diffusion of the R P is either too slow or too fast compared with the characteristic time constant of the recombination reaction, i.e., in low or high viscosity liquids or if Coulomb interaction keeps the RP in the reaction zone during a sufficiently long time.

Mathematically the dynamics of the exponential model are represented by equation (4) if one sets $\hat{\mathscr{B}}=-k_{\text {ce }}$, where $k_{\text {ce }}$ is the rate constant of 'cage escape', and takes $k_{\text {bet }}$ in the $\hat{W}$ operator as a constant. For the spin Hamiltonian and relaxation operator specified in equations (5) and (8), respectively, the exponential model can be solved analytically. A solution has been provided by Lüders and Salikhov [76]. However, there are some sign inconsistencies in their kinetic equations, and their final analytical result for the recombination probability is not in full accord with the analytical solution that we obtained with the help of 'Maple', a mathematical computation program [77, 78]. On the other hand our analytical results are in excellent agreement with our numerical solution of the exponential SLE whereas the Lüders and Salikhov formula ([76], equation (39)) gives systematic albeit only slight $(\approx 0.1 \%)$ deviations from those values. Here we provide the approved version of the analytical solution for the $S-T_{0}$ case:

$$
\varphi_{\mathrm{ce}}(\omega)=k_{\mathrm{ce}} \operatorname{tr}(\hat{\sigma})=\frac{1}{1+\frac{k_{\mathrm{bet}}}{k_{\mathrm{ce}}} q(\omega)},
$$

where

$$
\begin{array}{r}
q(\omega)=\frac{\omega^{2} T^{2}+T\left(2 k_{\mathrm{ce}}+k_{\mathrm{bet}}\right)+4}{2 \omega^{2} T^{2}+T\left(T\left(k_{\mathrm{ce}}+k_{\mathrm{bet}}\right)+4\right)} \\
\quad \times\left(2 k_{\mathrm{ce}}+k_{\mathrm{bet}}\right)+2 k_{\text {bet }} T+8
\end{array}
$$

is the factor describing the effect of spin control on the recombination. It may be interpreted as a correction factor to the BET rate constant $k_{\text {bet. }}$. The case of $q=1$ corresponds to the traditional (spin-less) exponential model. In the limit of high field $q=1 / 2$, which is equivalent to a spin-less system with $k_{\text {bet }}$ being replaced by $k_{\text {bet }} / 2$. The obvious interpretation of this limit is that $S$ and $T_{0}$ sublevels are fully equilibrated while the system can only recombine from the $S$ level.

The MFE on the cage escape efficiency is defined as

$$
M(\omega)=\frac{\varphi_{\mathrm{ce}}(\omega)-\varphi_{\mathrm{ce}}(0)}{\varphi_{\mathrm{ce}}(0)} .
$$

Combining equations (23)-(25) yields

$$
M(\omega)=-\frac{\omega^{2} T^{3} k_{\mathrm{bet}}\left(k_{\mathrm{ce}}+k_{\mathrm{bet}}\right)}{\left(\left(k_{\mathrm{ce}}+k_{\mathrm{bet}}\right) T+2\right) \Delta}
$$

with 


$$
\begin{aligned}
\Delta= & \left(2 k_{\mathrm{ce}}+k_{\text {bet }}\right)\left(\omega^{2} T^{2}+T^{2} k_{\mathrm{ce}}\left(k_{\mathrm{ce}}+k_{\text {bet }}\right)\right. \\
& \left.+{ }_{T}\left(4 k_{\mathrm{ce}}+k_{\text {bet }}\right)+4\right)+2 k_{\mathrm{ce}} k_{\text {bet }} T .
\end{aligned}
$$

\section{Results and discussion}

Figure 1 $(a)$ shows the experimentally observed MFE [67] on the efficiency $\varphi_{\text {ce }}$ of escape into the bulk of the solution of geminate R Ps created by reaction (3). The general characteristics of the MFE is a decrease in $\varphi_{\text {ce }}$ with the field with a decreasing slope. However, saturation is not yet reached at $3.2 \mathrm{~T}$, the highest field applied in these experiments. Qualitatively, the negative sign of the observed MFE, i.e., the decrease in $\varphi_{\text {ce }}$ with increasing field, is due to the spin-forbidden nature of the BET. Because the RP is created with the triplet multiplicity of its electronically excited precursor state, and the spin-mixing effect of a magnetic field based on the $g$ tensor difference of the two radicals accelerates the $T \rightarrow S$ process, the field enhances the reactivity in the BET process, which leads to a reduction in $\varphi_{\text {ce }}$. In the experiment, the solvent viscosity $\eta$ was varied by utilizing mixtures of $\mathrm{H}_{2} \mathrm{O}: \mathrm{ACN}(1: 1)$ with ethylene glycol. The viscosity variation ranged over more than a decade and can be seen from figure 1(b), where the effects observed for a given field relative to zero field are plotted versus viscosity. As can be seen in figure 1( $a)$, the amplitude of the MFE curve is affected significantly by a change in solvent viscosity. An increase of viscosity tends to enhance the (negative) MFE at low fields, whereas this tendency is reversed at high fields. This viscosity dependent behaviour of the MFE is shown in a different representation as $\varphi_{\text {ce }}$ versus $\log (1 / \eta)$ in figure $1(b)$, which gives a clearer picture since curve crossings are avoided. What is, quite conspicuously, borne out in this representation is that for any field the MFE passes an extremum at intermediate viscosities. Concurrent with an increase in absolute value, the position of the extremum shifts to higher values of $1 / \eta$ as the field is increased. For the highest field the low viscosity branch of the curve is cut off at the lowest viscosity experimentally explored.

The curves fitting the experimental points in figure 1(a) resulted from theoretical calculations based on the exponential model [67]. These calculation s employed a spin formalism with the full $4 \times 4$ spin densitiy matrix. The fits, which reproduce the shape of the MFE curves very well, were used to determine specific values of $T$, $k_{\text {ce }}$, and $k_{\text {bet }}$ for each solvent viscosity, whereupon it was found that $T$ is rather insensitive to $\eta, k_{\mathrm{ce}}$ varies approximately linearly with $1 / \eta$ and $k_{\text {bet }}$ also shows a pronounced covariance with $1 / \eta$, albeit not a linear one. While the first two findings were expected (spin relaxation in the $\mathrm{Ru}$ complexes is determined by an intramo- lecular spin-orbit coupling based mechanism, i.e., has scarcely any contribution from viscosity dependent mechanisms related to molecular rotation [58, 79], whereas cage escape is the prototype of a diffusion dependent process), a solvent viscosity dependence of $k_{\text {bet }}$ is unexpected within the standard theory of nonadiabatic electron transfer, if solvent reorganization energy and electronic coupling matrix element remain unaffected. Since there is no reason to assume that these latter parameters are changed significantly in the series of solvent mixtures employed it was argued that the observed variation of the $k_{\text {bet }}$ parameter might indicate the presence of a pronounced dynamic solvent effect on the BET rate.

The present theoretical investigation based on the diffusional model does not attempt to simulate the MFE curves of figure $1(a)$ in all details. Rather, our aim here is to study the principal predictions about the viscosity dependence of the MFE on the basis of the diffusional model combined with the assumption that nonadiabatic, Marcus-type electron transfer with viscosity independent electron transfer rate parameters prevails throughout the solvent variation. The diffusion constant will be the only parameter representing the change in solvent properties. Furthermore, these investigations can serve also as a principal test to decide the limits within which the exponential model is physically consistent. The predictions of the exponential model for a purely diffusional viscosity effect will be simulated by a variation of $k_{\mathrm{ce}}$ only.

For the viscosity-independent parameters in the diffusional model the following values were used: ${ }_{a}=10 \AA, T=15 \mathrm{ps}, W_{0}=300 \mathrm{~ns}^{-1}$, and $L=0.75 \AA$. The values of $a$ and $T$ represent the correct order of magnitude for the $\mathrm{Ru}(\mathrm{bpy}){ }_{3}{ }^{2+} / \mathrm{MV}^{2+}$ type of electron transfer system. For FET and BET the same electron transfer parameters $W_{0}$ and $L$ were used. $L=0.75 \mathrm{~A}$ is in the typical range of values found in the literature $[6$, $10,11,24,26]$. The value of $W_{0}$ was fixed to yield the correct order of magnitude of the observed $\varphi_{\text {ce }}$, which varies between 0.2 and 0.05 over the range of viscosities investigated [67]. It may be noted here that this range of values for $\varphi_{\mathrm{ce}}$ indicates that recombination is in the diffusion controlled regime [75]. For the forward reaction the realistic value of $W_{0}$ is probably lower, but for the sake of simplicity and since the character of the present study is a rather qualitative one we did not give it a different value. For the same reasons we did not take into account explicitly the effects of an interparticle potential $V(r)$, although reactants and radicals of the reaction system (3) are charged and exhibit Coulombic interactions. Since the experiments were carried out in solvents of high polarity and with high ionic 
strength, it appears that this neglect is not a severe approximation.

For our calculation with the exponential model, $T$ was also fixed to $15 \mathrm{ps}$. The value for the exponential $k_{\text {bet }}$, denoted $k_{\text {bet }}^{\prime}$ for distinction, was determined such that for $D=10^{-5} \mathrm{~cm}^{2} \mathrm{~s}^{-1}$ the spinless exponential model yielded the same value of the cage escape efficiency $\varphi_{\text {ce }}$ as a model with diffusion and spin-indepen dent reaction on contact. This was achieved by setting:

$$
k_{\text {bet }}^{\prime}=k_{\mathrm{ce}} \frac{1-\varphi_{\mathrm{ce}}}{\varphi_{\mathrm{ce}}}
$$

with

$$
k_{\mathrm{ce}}=\frac{3 D}{a^{2}},
$$

which provides the most consistent connection between the two models [75].

Figure 2 shows the theoretical predictions following from the exponential model for the $k_{\text {ce }}$ depencence of the escape $\eta_{c e}$ efficiency at different $S / T$ mixing rates $\omega$. Note that according to equation (2) and for an average value of 1 for $\Delta g$ in the systems considered, an angular frequency of $100 \mathrm{rad} \mathrm{ns}^{-1}$ corresponds to a field of about $1 \mathrm{~T}$. For any mixing frequency $\omega$ the curves for the pertinent MFE $M$ (cf. figure $2(b)$ ) exhibit a monotonic decrease with $k_{\mathrm{ce}}$, starting with zero at high $k_{\mathrm{ce}}$, i.e., in the limit of low viscosity, and approaching a saturation value at $k_{\mathrm{ce}} \rightarrow 0$, i.e., in the limit of high viscosity. For a finite mixing frequency $\omega$, the behaviour in different limits is described by the following expressions:

$$
\lim _{k_{\mathrm{ce}} \rightarrow 0} M(\omega)=-\frac{\omega^{2} T^{3} k_{\text {bet }}}{\left(k_{\text {bet }} T+2\right)\left(\omega^{2} T^{2}+k_{\text {bet }} T+2\right)},
$$

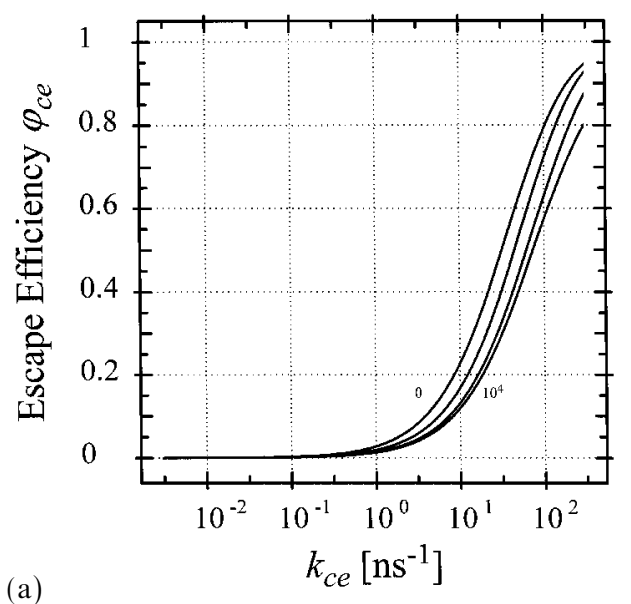

$$
M(\omega) \propto-\frac{\omega^{2} k_{\mathrm{bet}}}{2 k_{\mathrm{ce}}^{3}} \quad \text { for } \omega, k_{\mathrm{bet}}, \frac{1}{T} \ll k_{\mathrm{ce}},
$$

while for any $k_{\text {ce }}$ the high field limit of $M(\omega)$ is given by

$$
\lim _{\omega \rightarrow \infty} M(\omega)=-\frac{k_{\text {bet }}\left(k_{\text {bet }}+k_{\mathrm{ce}}\right)_{T}}{\left(k_{\text {bet }}+2 k_{\text {ce }}\right)\left(k_{\text {bet }}+k_{\text {ce }}\right)_{T}+2} .
$$

Comparing figures $2(b)$ and $1(b)$ it is clear that the exponential model cannot account for the characteristic minima in the viscosity dependence of the MFE curves, if all parameters except $k_{\mathrm{ce}}$ are kept constant.

In figure 3 the corresponding results for the diffusional model are shown. Here, the diffusion constant $D$ is the only viscosity dependent parameter. While the general behaviour of the cage escape efficiency $\varphi_{\text {ce }}$ versus $D$ is qualitatively similar to what one obtains with the exponential model, where $k_{c e}$ is varied instead of $D$ (cf. figures $2(a)$ and $3(a))$, the curves for the magnetic field effect $M(\omega)$ (cf. figures $2(a, b)$ ) now display characteristically different behaviour with minima (i.e., maxima of the field effect amplitude) at intermediate viscosities, shifting to lower viscosities with increasing mixing frequency $\omega$. In fact, for the approximate range of parameters corresponding to the experimentally scanned range of $D$ (cf. dashed box in figure $3(b)$ ) the calculated curves exhibit approximately the same behaviour as the observed ones shown in figure 1 $(b)$. Thus it appears that the observed viscosity dependence of the MFE curves do indeed reflect specific features of the diffusional model that are not present in the exponential model. In the following we will try to identify those particular features of the diffusional model that are responsible for the characteristic minima in the MFE versus viscosity curves.

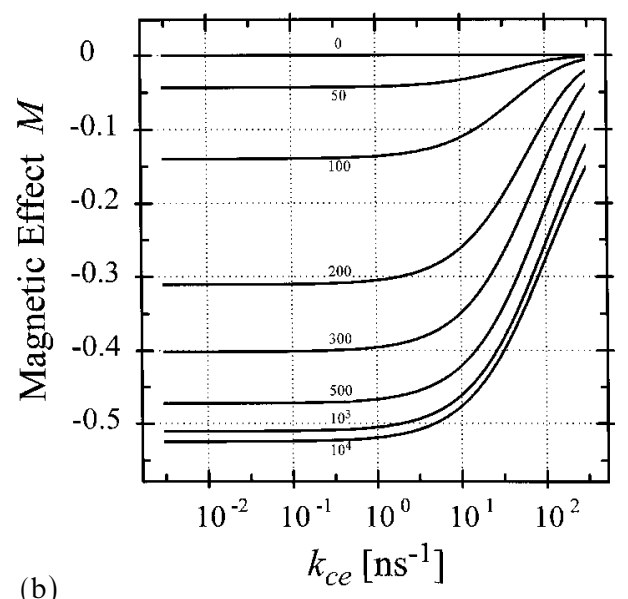

(b)

Figure 2. Exponential model: $(a)$ cage escape efficiency $\varphi_{\mathrm{ce}}$ as a function of the separation rate constant $k_{\mathrm{ce}}$ (parameters of calculation, $k_{\text {bet }}=147.1 \mathrm{~ns}^{-1}, T=15 \mathrm{ps}, \omega$ (from left to right) $0,200,500$ and $10^{4} \mathrm{rad} / \mathrm{ns}$ ); $(b)$ relative MFE $M$ on $\varphi_{\mathrm{ce}}$ (the numbers assigned to the curves denote the frequency $\omega$ (in $\mathrm{rad} \mathrm{ns}^{-1}$ ) of $s-T_{0}$ conversion). 

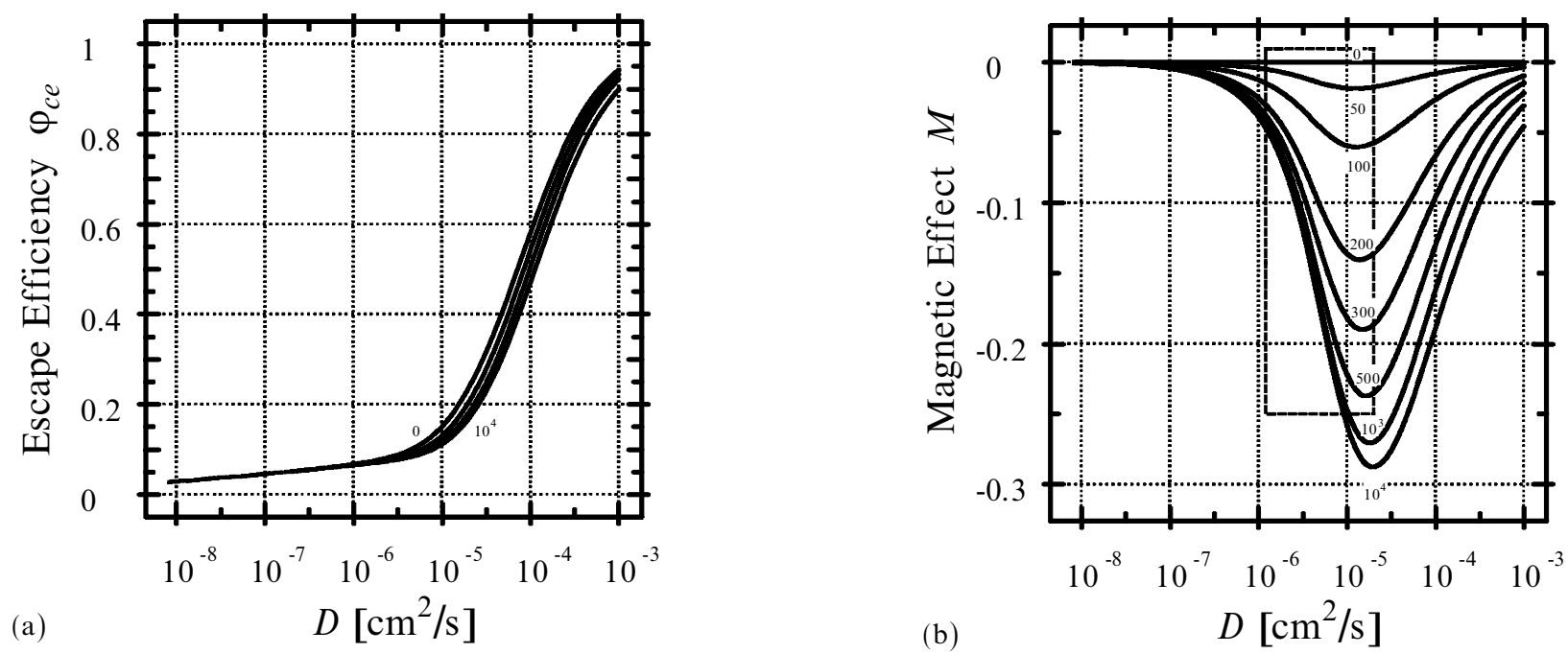

Figure 3. Diffusional model: $(a)$ cage escape efficiency $\varphi_{\mathrm{ce}}$ as a function of the diffusion constant $D$ (parameters of calculation, $a=10 \mathrm{~A}, \tau_{\mathrm{D}}=1210 \mathrm{~ns}, c_{\mathrm{Q}}=0.1 \mathrm{M}, W_{0}=300 \mathrm{~ns}^{-1}, L=0.75 \mathrm{~A}, T=15 \mathrm{ps}, \omega$ (from left to right) $0,200,500$, and $10^{4} \mathrm{rad} \mathrm{ns}^{-1}$ ); (b) relative MFE on $\varphi_{\text {ce }}$ (the numbers assigned to the curves denote the frequency $\omega$ of $S-T_{0}$ conversion). The rectangle in dashed lines indicates the region approximately spanned by the experimental data in figure $1(b)$.

In the diffusional model the interradical distance $r$ in the R Ps is a crucial variable. As described in section 2 we consider the average of $\varphi_{\text {ce }}$ over an ensemble of R Ps that are created with some $r_{o}$ distribution depending on the characteristics of the forward electron transfer reaction. Instead of performing the average over this distribution it may be instructive to consider results of the diffusional model for specific initial distances $r_{o}$ of the RP. Thus we now substitute the initial densitiy matrix $\hat{\rho}_{0}(r)$ obtained from equation (20) and describing a more or less extended initial distribution by a spin density matrix $\hat{\theta}_{0}(r)$ corresponding to a distribution localized at $r_{0}$ :

$$
\hat{\theta}_{0}(r)=\frac{\delta\left(r-r_{0}\right)}{4 \pi_{0}^{2}}\left(\begin{array}{ll}
0 & 0 \\
0 & 1
\end{array}\right) .
$$

Figure 4 shows the $\varphi_{\text {ce }}$ and MFE versus $D$ curves for $R$ Ps with different initial separations. As follows from these figures, for a given $D$ the cage escape efficiency $\varphi_{\text {ce }}$ increases and the MFE amplitude decreases with increasing initial separation. This behaviour is of course expected since it is clear that the chance for escape is larger when the R P starts at a larger $r_{0}$ and the magnetic field can exhibit its effect only in so far as BET can take place. Concerning the behaviour of the individual MFE curves in figure 4(b), the most striking feature is the occurrence of minima seen clearly at $r_{0}>11 \mathrm{~A}$ and still there as shoulders at $r_{0}<11 \mathrm{~A}$. The minima move to smaller absolute MFEs and to higher $D$ values, i.e., lower viscosities, with increasing $r_{0}$. At very low $D$ values, i.e., high viscosities, the MFE curves turn down and approach limiting values depending on $r_{0}$. (this limiting behaviour is not shown in figure $4(b))$. It should be noted that the final high viscosity branch of the curves falls in a region where $\varphi_{\mathrm{ce}}<0.01$ (cf. dotted line in figure $4(b)$ ), i.e., where MFEs on $\varphi_{\text {ce }}$ will be difficult to measure due to experimental sensitivity limits.

Phenomenologically, the $D$ dependence of the MFE curves may be divided into three regimes I, II, III, where, passing from high to low $D$ values, the slope of the MFE curve is positive, negative, positive. In order to rationalize this non-monotonic behaviour let us introduce a length parameter

$$
d_{\text {rel }}=(D T)^{1 / 2},
$$

scaling the average distance of diffusion the R $\mathrm{P}$ will undergo during the spin relaxation time $T$. The characteristic MFE behaviour in the three regimes may be rationalized employing this length parameter and the following heuristic rule: 'If the spatial distribution does not change substantially during the time span in which most of the spin evolution and reaction occurs, the viscosity dependence of the MFE will be qualitatively the same as predicted by the exponential model. It will be opposite to that if spin evolution is going on while the radicals diffuse to their preferred distance of reaction'.

Based on the diagrams in figure 5 , we will now give a demonstration of this basic rule by comparing for a given $r_{0}$, e.g., $11.5 \mathrm{~A}$, and for six selected values of $D$ the MFE as represented in figure $4(b)$ with the distribution of the recombination probability, $\varphi_{\text {rec }}\left(r \mid r_{0}\right)$, defined as 

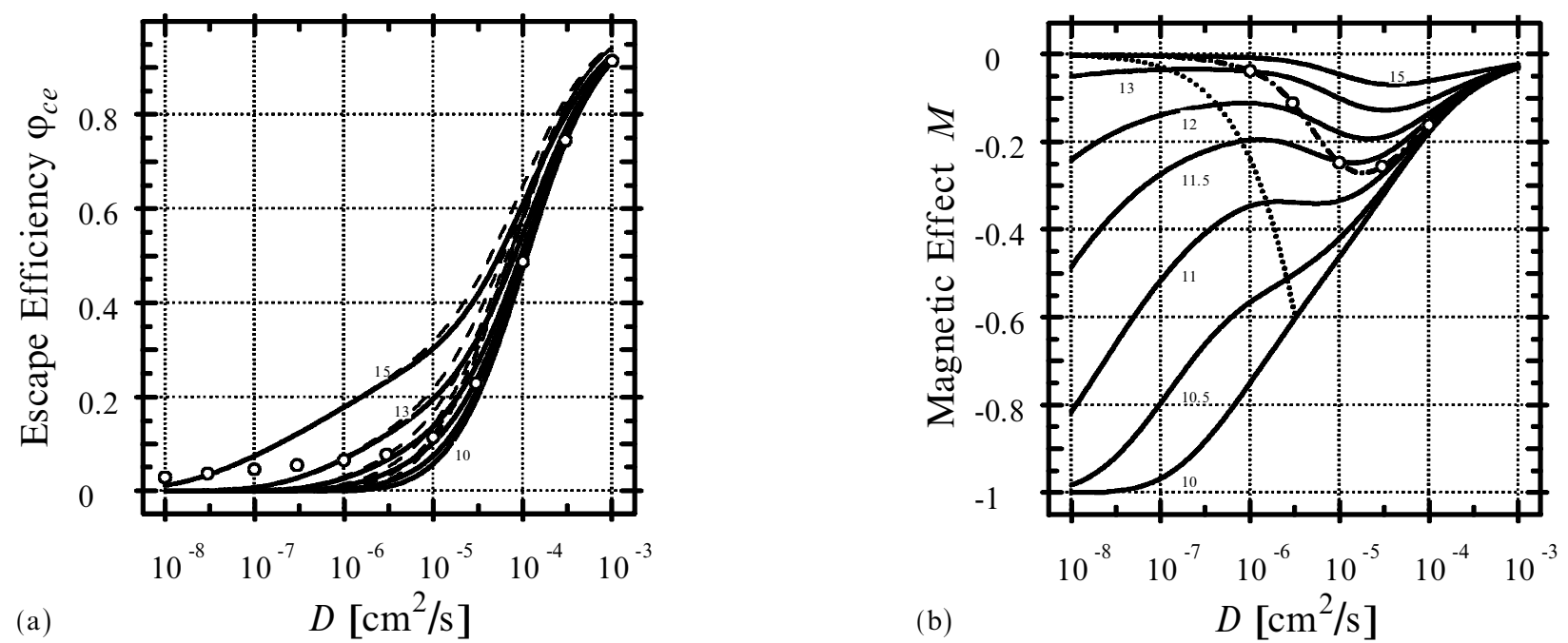

Figure 4. Diffusional model: $(a)$ cage escape efficiency $\varphi_{\text {ce }}$ as a function of the diffusion constant $D$ for R Ps with specific initial separation. Initial separations $r_{0}$ (from right to left): 10, 10.8, 11.4, 12, 13 and $15 \mathrm{~A}$, frequency of $S-T_{0}$ transitions $\omega=1000 \mathrm{rad} \mathrm{ns}^{-1}$ (solid lines) and $\omega=0$ (dashed lines). The other parameters of the calculation are the same as for the curves in figure 3. The open circles represent the curve for $\omega=1000 \mathrm{rad} \mathrm{ns}^{-1}$ from figure 3(a) where the distribution of initial distances is determined by the forward electron transfer kinetics. $(b)$ MFE ourves corresponding to the $\varphi_{\mathrm{ce}}$-values in part $(a)$. The numbers at the curves denote the initial separation distance $r_{0}$ in A. The dash-dotted line represents the $\omega=1000 \mathrm{rad} \mathrm{ns}^{-1}$ curve from figure $3(b)$. The circles on this curve represent certain situations that will be explored further in figure 6 . The dotted line connects points with a constant $\varphi_{\mathrm{ce}}$ value of 0.01 . In the region to the left of this curve the $\varphi_{\mathrm{ce}}$ values are less than 0.01 , and to the right they are higher.

$$
\begin{aligned}
\varphi_{\text {rec }}\left(\left.r\right|_{r_{0}}\right) & \equiv 4 \pi^{2} \operatorname{tr}\left(\int_{0}^{\infty} \frac{1}{2}\left[\hat{W}(r), \hat{\rho}\left(\left.r\right|_{r_{0}}, t\right)\right]_{+} \mathrm{d} t\right) \\
& =2 \pi^{2} \operatorname{tr}\left[\hat{W}\left(r_{\text {rec }}\right), \hat{\sigma}\left(r_{\text {rec }} \mid r_{0}\right)\right]_{+}
\end{aligned}
$$

For R Ps created with initial separation $r_{0}, \varphi_{\text {rec }}\left(r \mid r_{0}\right) \mathrm{d} r$ denotes the portion recombining in the interval $r$ to $r+\mathrm{d} r$. As may be noted, $\varphi_{\text {rec }}\left(\left.r\right|_{r_{0}}\right)$ is an analogue of the initial distribution $f_{0}(r) \equiv 4 \pi^{2} \operatorname{tr}\left(\hat{\rho}_{0}(r)\right)$. The latter is interpreted as a distribution of probability for R Ps created at distance $r$ in the course of FET reaction. As was shown in [40], the distribution $f_{0}(r)$ adopts different shapes in the three different FET regimes: in the kinetic regime, $f_{0}(r)$ replicates $4 \pi^{2} k_{\text {fet }}(r)$; in the diffusion-controlled regime, $f_{0}(r)$ has a bell-shaped form with a maximum located approximately at a separation $R$ eff defined by the relation $k_{\mathrm{q}}=4 \pi R_{\text {eff }} D$ established for the stationary value of the diffusion controlled rate constant of FET; and in the static regime, $f_{0}(r)$ is a wide distribution, decreasing monotonically with $r$.

As will be shown in the following, the same regimes determine also the shape of $\varphi_{\mathrm{rec}}\left(\left.r\right|_{r_{0}}\right)$, and are reflected in the viscosity dependence of the MFE on the separation efficiency $\varphi_{\mathrm{ce}}$.

Regime I. (Positive slope of MFE versus $D$ ): kinetically controlled recombination (cf. cases $D=10^{-4}$ and $3 \times 10^{-5} \mathrm{~cm}_{0}^{2} \mathrm{~s}^{-1}$ in figure 5). In these cases $d_{\text {rel }}=3.87 \mathrm{~A}$ or $2.12 \mathrm{~A}$ is larger than the length par- ameter $L$ of the distance-dependence of $k_{\text {bet }}$ and larger than the length $r_{0}-a=1.5 \mathrm{~A}$ separating the created R P from contact. From the validity of the condition $d_{\text {rel }}>\min \left(L, r_{0}-a\right)$ it follows that diffusion may establish a rather uniform distribution of R Ps over a reaction zone extending from $a$ over several units of $L$ before the $T \rightarrow S$ process is completed in zero field. Hence spinequilibration will occur rather uniformly over all the positions of this distribution, and the distribution of reaction distances will closely match the $r$ dependence of $k_{\text {bet }}(r)$. Thus it is the electron transfer kinetics rather than the diffusion which control recombination. From the rule stated above it follows that in regime $I$ the change of the MFE with viscosity should be similar to that predicted by the exponential model.

Regime II. (Negative slope of MFE versus $D$ ): diff usion controlled recombination ( $\mathrm{cf}$. cases $D=10^{-5}, 3 \times 10^{-6}$, $10^{-6} \mathrm{~cm}^{2} \mathrm{~s}^{-1}$ in figure 5). In this regime $d_{\text {rel }}(=1.22,0.67$, $0.39 \AA)$ is of the same order as $L$ and equal to or smaller than $r_{0}-a$, i.e., subject to the condition $L \approx d_{\text {rel }} \leq_{0} r_{0}-a$. Actually the cases $d_{\text {rel }}=2.2 \AA \approx 2 L$ and $0.39 \mathrm{~A} \approx L / 2$ should be considered as borderline cases. In fact, for these latter values the particular MFE curve considered is close to its minimum and maximum, respectively. The range of $d_{\text {rel }}$ values specified for regime II implies that the distribution of R P distances characteristic of regime I cannot be established before spin equilibrium is reached, which means that 


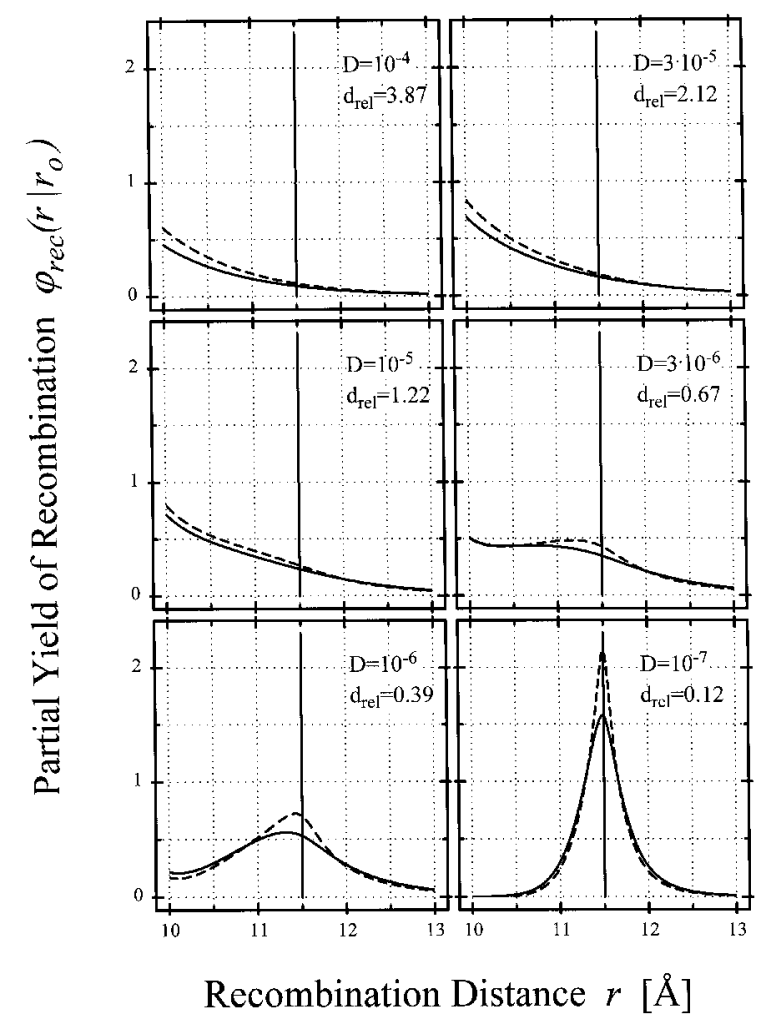

Figure 5. Distribution function $\varphi_{\text {rec }}\left(r_{\text {rec }} \mid r_{0}\right)$ (cf. equation (35)) for R Ps created at $r_{o}=11.5 \mathrm{~A}$ and recombining at $r_{\text {rec }}$. The vertical bars mark the initial separation of 11.5 A. Solid lines, reaction in zero field; dashed lines, reaction in a field corresponding to $\omega=1000 \mathrm{radns}^{-1}$. The value of $D$, the diffusion coefficient (in $\mathrm{cm}^{2} \mathrm{~s}^{-1}$ ), and the corresponding relaxation length $d_{\text {rel }}$ (in A) resulting from equation (33) are given in each of the diagrams.

spin evolution occurs while the $\mathrm{RP}$ is on the way to positions of higher reactivity. As can be seen, for the values of $D$ referred to, the deviation of the distribution $\varphi_{\text {rec }}\left(r_{\text {rec }} \mid r_{0}\right)$ from the exponential $r$ dependence of $k_{\text {bet }}(r)$ becomes more and more pronounced as $D$ decreases. We can even observe formation of a maximum at $r_{\text {rec }}>a$ moving away from contact as $D$ decreases. This means that the recombination is limited by the delivery of R Ps to the region of efficient recombination, i.e., it is under diff usion control.

Regime III. (Positive slope of MFE versus $D$ ): static recombination. (cf. case $D=10^{-7} \mathrm{~cm}^{2} \mathrm{~s}^{-1}$ ). Here one observes that the maximum of the distribution $\varphi_{\text {rec }}\left(r_{\text {rec }} \mid r_{0}\right)$ is very close to $r_{0}$, i.e., the diffusional motion is too slow to adapt the distribution to the exponential $r$ dependence of $k_{\text {bet }}(r)$ during the spin relaxation time $T$. Essentially, the R Ps react where they were born. This is due to the fact that $d_{\text {rel }} \ll \min \left(L, r_{0}-a\right)$, which hence marks the condition for regime III. Here, as in regime I but for a different reason, we are again facing the situation that spin evolution and reaction do occur over spatial distributions of R Ps that are rather independent of viscosity. So, according to the heuristic rule, this should again lead to a viscosity dependence of the MFE with the same sign of slope as expected from the exponential model. This is indeed the case. Furthermore, we note that for $D \rightarrow 0$ the MFE versus $D$ curves approach limiting values which coincide exactly with the limiting MFEs derived from the exponential model for $k_{\mathrm{ce}} \rightarrow 0$ (cf. equation (21) and figure $2(b)$ ) if one uses $k_{\text {bet }}^{\prime}=k_{\text {bet }}\left(r_{0}\right)$.

When comparing the MFE versus $D$ curves for different $r_{0}$ we note that the minima (i.e., the borders between regimes I and II) and the maxima (i.e., the borders between regimes II and III) come closer as $r_{0}$ decreases. This is quite natural in terms of the above presented reasoning, because as $r_{0} \rightarrow a$ regimes $\mathrm{I}$ and III expand towards each other and the range for regime II becomes narrower. Finally, the branch with negative slope of the MFE versus $D$ curve disappears. The curve for $r_{0}=10.6 \mathrm{~A}$ is close to this borderline case. For smaller $r_{0}$ the curves still exhibit an inflection point in the region where regimes I and III merge, which is a persisting indication of the influence of regime II type behaviour. This also may be interpreted as disappearance of the diffusion-controlled regime because the closer to contact the R Ps are born, the smaller is the influence the transport process has on recombination.

In figure $4(b)$ we have indicated the MFE versus $D$ curve from figure $3(b)$ obtained for the same mixing frequency $\omega$. This curve resulted from considering a distribution of $r_{0}$ values following from the proper treatment of diffusion- and distance-dependent forward electron transfer in the quenching of the excited state. It appears that this line shows much more pronounced regime II behaviour than the curves with discrete $r_{0}$. For any distribution over $r_{0}$ we may define an effective $r_{0}$ denoted as $r_{0, \text { eff }}$, by assigning it that $r_{0}$ value which would yield the same MFE at the actual $D$ value. (The consistency of this definition is supported by the fact that approximately the same $r_{0, \text { eff }}$ values ensue if one defines it by applying the corresponding criterion to the curves of $\varphi_{\text {ce }}$ versus $D$ (cf. figure $4(a)$ ). From the behaviour of the dash-dotted line in figure $4(b)$ we can conclude that, for the RP ensemble created in the FET reaction, $r_{0, \text { eff }}$ increases with increasing viscosity. This type of phenomenon has been investigated theoretically in detail by Burshtein and coworkers [40]. The general results obtained by them may be illustrated by figure 6 , showing our numerical results for the $f_{0}(r)$ profiles with the present model system: in the low viscosity limit (kinetically controlled FET), the pair distribution function profile $n(r, t)$ (see equations $(15)-(17))$ is not affected by electron transfer, $n(r, t) \cong 1$, and therefore 


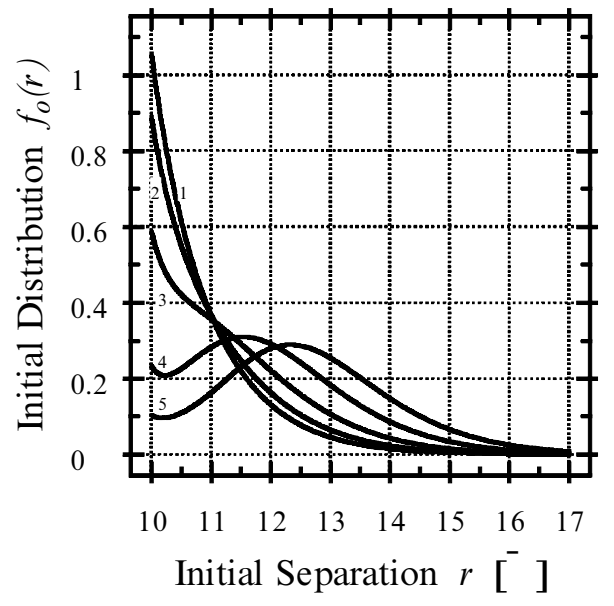

Figure 6. Effect of viscosity on initial distribution $f_{0}(r) \equiv 4 \pi^{2} \operatorname{tr}\left(\hat{\rho}_{0}(r)\right)$ obtained by treating the FET reaction in the diffusional model. The pertinent values of the diffusion coefficient $D$, in $\mathrm{cm}^{2} \mathrm{~s}^{-1}$ are: $1,10^{-4} ; 2,3 \times 10^{-5}$; $3,10^{-5} ; 4,3 \times 10^{-6}$; and $5,10^{-6}$. The other parameters are the same as in figure 3 . It should be noted that with the quencher concentration of $0.1 \mathrm{M}$ used the distribution is not significantly different from one resulting in the limit of $c_{\mathrm{Q}} \rightarrow 0$.

the source term $\hat{s}(r, t)$ (equations (4) and (11)) reproduces the distance dependence of $k_{\text {fet }}(r)$, the rate constant of FET (cf. equation (14)). For high viscosities the FET is controlled by diffusion, and the $r_{0}$ distribution $f_{0}(r)$ is built up in two stages. The first one corresponds to the non-stationary regime of the FET reaction, when the pair distribution function of reactants $n(r, t)$ changes from $n(r, t=0)=1$ to a stationary function $n_{\mathrm{s}}(r)={ }_{n}(r, t \rightarrow \infty)$. In the beginning of this stage, the source term $\hat{s}(r, t)$ reproduces the shape of $k_{\mathrm{fet}}(r)$ exactly, as in the kinetically controlled regime. This contributes mainly to the 'contact' part of $f_{0}(r)$. By the end of the first stage and during the second one the pair distribution profile $n_{\mathrm{s}}(r)$ is no longer uniform but decreases strongly in the vicinity of the contact radius $a$. During the second stage (which lasts until all excited donors have been quenched $\left({ }_{N}(t)=0\right.$, equation (13)) the non-contact part of $f_{0}(r)$ is formed and therefore the source term $\hat{s}(r, t)$ becomes bell-shaped. The higher the viscosity, the less is the contact contribution in the $n_{\mathrm{s}}(r)$ profile, and therefore the more distant is the centre of the $f_{0}(r)$ distribution from contact.

We have now identified and explained the two essential factors that lead to the characteristic regime II behaviour of the diffusional model. (i) For any given $r_{0}>a$, diffusion control of BET leads to a viscosity dependence of the MFE which is opposite to the case of distance-independent BET rate as in the exponential model. For fixed viscosity the MFE amplitude decreases as $r_{0}$ increases. (ii) Distance dependence of FET leads to

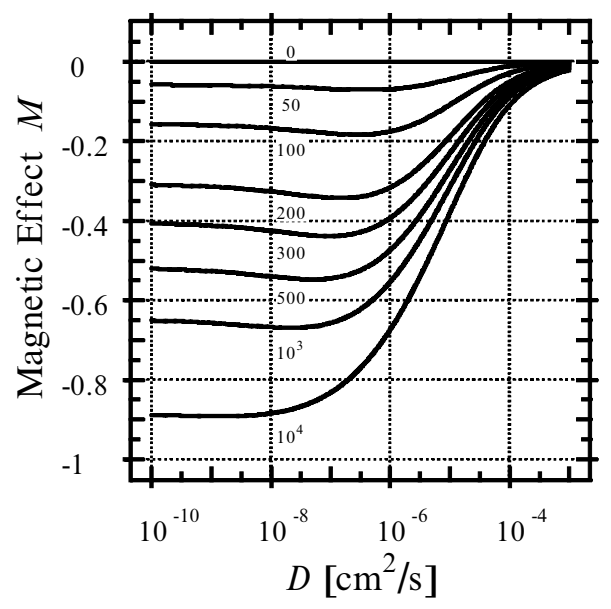

Figure 7. Magnetic field effect $M$ calculated for the diffusional model with formation and recombination of $\mathrm{RP}$ in contact only. The reactivity of singlet R Ps corresponds to recombination at first contact (Smoluchowski condition), $T=15 \mathrm{ps}$, and the numbers assigned to the curves denote the $S-T_{0}$ conversion frequency $\omega$ in $\mathrm{rad} \mathrm{ns}^{-1}$. The curves shown were obtained using the analytical expression derived in [73]. A value of 263.1 $\mathrm{A} \mathrm{ns}^{-1}$ was used for the parameter $\kappa$. Based on the relation $4 \pi a^{2} \kappa=$ $\int_{a}^{\infty} 4 \pi^{2} k_{\text {bet }}(r) \mathrm{d} r$ this value for $\kappa$ assures the same effective electron transfer rate constant as in our calculations with distance-dependent electron transfer.

increasing effective distance $r_{0 \text {,eff }}$ of the $\mathrm{RP}$ with increasing viscosity. This effect enhances factor (i), i.e., makes the viscosity dependence of the MFE steeper and extends the lower boundary of regime II behaviour to infinitely small $D$; thus it leads to the disappearance of regime III. Therefore it appears that the viscosity dependence of the MFE in regime II is really a fingerprint of the distance dependence of electron transfer.

As we have seen when analysing figure 5, the essential requirement of regime II behaviour is that spin motion takes place while the radicals are on their way to the reaction zone. It may be of interest in this regard that a weak indication of this effect can be demonstrated also for the case when both FET and BET occur at contact only. Applying the analytical solution of Mints and Pukhov [73] to our model system we obtained the MFE versus $D$ curves shown in figure 7. They do in fact exhibit shallow minima that might be considered as indicating the onset of a regime II behaviour. Such a behaviour might be assigned to the contribution of diffusional trajectories involving separation and reencounter, bringing the $\mathrm{R} P$ again into reactive contact after it separated and spent some time during which spin evolution could take place. However, as can be seen from figure 7, such re-encounter contributions to regime-II behaviour actually are negligible in comparison with the effects caused by distance-dependent FET and BET. 
As the present investigation has shown, the exponential model cannot account for the decrease of the MFE with increasing viscosity if the rate constant $k_{\text {bet }}$ is assumed to be viscosity independent. Therefore, in attempting to account for the experimentally observed feature on the basis of the exponential model, in the previously presented analysis [67] it had to be assumed that $k_{\text {bet }}$ is viscosity dependent, which led to the interpretation that in the solvents investigated the BET electron transfer in the $\mathrm{Ru}(\mathrm{bpy})_{3}^{3+} / \mathrm{MV}^{+\cdot}$ system is subject to a pronounced dynamic solvent effect. As demonstrated in the present paper, the diffusional model in combination with the distance-dependent rate constant of electron transfer can account qualitatively for the observed viscosity dependence of the MFE without assuming a viscosity dependence of the electron transfer rate constant itself. However, as was pointed out in the beginning, the aim of this paper was to focus on the principal aspects of how within a realistic model of diffusional motion the distance dependence of FET and BET is reflected in an MFE on the cage escape efficiency. Therefore, regarding the description of spin motion, the model was kept as simple as possible, although the employed parameter values were adapted to the situation in the real system. It will be a separate task to analyse the details of the experimental results as shown in figure 1 on the basis of a spin-chemically more detailed diff usional model, employing a full representation of the $4 \times 4$ spin space of the RP, accounting properly for $g$ tensor anisotropy, for the spin-orbit coupling perturbed nature of the spin substates and, as far as electron transfer is concerned, to allow for a specific difference in the rate constants of FET and BET. Such investigations are now in progress and it remains to be shown whether in a quantitative treatment with the diffusional model there is still the necessity to assume some viscosity dependence of electron transfer parameters, indicating the presence of a dynamic solvent effect. In any case, a final decision on this issue will require a ps time-resolved experimental investigation of fast electron transfer processes in the pertinent series of solvents, not necessarily with the $\mathrm{Ru}(\mathrm{bpy})_{3}^{2+} / \mathrm{MV}^{2+}$ systems.

\section{Conclusion}

It has been established that MFEs represent sensitive probes of the details of the electron transfer mechanism in photoinduced radical formation. In fact, although the viscosity dependence of $\varphi_{\text {ce }}$ predicted from the diffusional model with distance-dependent electron transfer (cf. figure $3(a)$ ) and that predicted by the simpler exponential model (cf. figure $2(a)$ ) are similar, a drastic qualitative difference is found when the MFE predictions of the two models are compared (cf. figures $2(b)$ and $3(b))$. Thus the MFE versus viscosity curves can serve as a true fingerprint of the role of diffusion- and distance-dependent electron transfer in the photoinduced generation of free radicals.

Finally, it should be pointed out that the magnetic controllability of spin evolution - and connected with it: reactivity - on the ps timescale represents an important requirement of R P systems suitable for experimentally probing such effects. Therefore, systems with large $\Delta g$ are required, which makes $\mathrm{R} P$ s with paramagnetic metal complexes (d electron radicals) particularly attractive.

This work was supported by the Volkswagen-Stiftung within the priority area Inter- and Intramolecular Electron Transfer and by the Isreali Science Foundation. Grants to N.N.L. by the European Community (INTAS 93-1626-EXT) and by the Russian Foundation of Basic Researches (RFBR Grant 98-03-33180) are gratefully acknowledged. Special thanks are due to the Alexander-von-Humboldt foundation for fellowship grants to N.N.L. and E.B.K.

\section{References}

[1] Khairutdinov, R. F., Zamarev, K. I., and Zhadanov, V. P., 1989, Comprehensive Chemical Kinetics, Vol. 30, edited by R. G. Compton (Amsterdam: Elsevier).

[2] Kagan, Yu., and Lescett, A. J., 1992, Modern Problems in Condensed Matter, Vol. 34, edited by V. M. Agranovic and A. A. Maradudin (Amsterdam: North-Holland).

[3] Roy, D. K., 1986, Quantum Mechanical Tunnelling and Its Applications (Singapore: World Scientific).

[4] Closs, G. L., and Miller, J. R., 1988, Science, 240, 440.

[5] Logan, J., and Newton, M. D., 1983, J. chem. Phys., 78, 4086.

[6] Marcus, R. A., and Sutin, N., 1985, Biochimc. Biophys. Acta, 811, 265.

[7] Isied, S. S., Vassilian, A., Wishart, J. F., Creutz, C., Schwarz, H. A., and Sutin, N., 1988, J. Amer. chem. Soc., 110, 635.

[8] Tachiya, M., and Murata, S., 1992, J. phys. Chem., 96, 8441.

[9] Burshtein, A. I., Frantsuzov, P. A., and Zharikov, A. A., 1991, Chem. Phys., 155, 91.

[10] Murata, S., Matsuzaki, S. Y., and Tachiya, M., 1995, J. phys. Chem., 99, 5354.

[11] Kakitani, T., Matsuda, N., Yoshimori, A., and Mataga, N., 1995, Progr. Reaction Kinetics, 20, 347.

[12] Meier, M., van Eldik, R., Chang, I.-J., Mines, G. A., Wuttke, D. S., Winkler, J. R., and Gray, H. B., 1994, J. Amer. chem. Soc., 116, 1577.

[13] Oliver, A. M., Craig, D. C., Paddon-Row, M. N., Kroon, J., and Verhoeven, J. W., 1988, Chem. Phys. Lett., 150, 366.

[14] Mataga, N., Asahi, T., Kanda, Y., Okada, T., and Kakitani, T., 1988, Chem. Phys., 127, 249.

[15] Kumar, K., Lin, Z., Waldeck, D. H., and Zimmt, M. B., 1996, J. Amer. chem. Soc., 118, 243.

[16] Miller, J. R., 1975, Science, 189, 221.

[17] Beitz, J. V., and Miller, J. R., 1979, J. chem. Phys., 71, 4579. 
[18] Guarr, T., McGuire, M., Strauch, S., and Mclendon, G., 1983, J. Amer. chem. Soc., 105, 616.

[19] Miller, J. R., Calcaterra, L. T., and Closs, G. L., 1984, J. Amer. chem. Soc., 106, 3047.

[20] Closs, G. L., Calcaterra, L. T., Green, N. J., Penfield, K. W., and Miller, J. R., 1986, J. phys. Chem., 90, 3673.

[21] Wasielewski, M. R., Niemczyk, M. P., Johnson, D. G., Svec, W. A., and Minsek, D. W., 1989, Tetrahedron, 45, 4785.

[22] Oevering, H., Paddon-Row, M. N., Heppener, M., Oliver, A. M., Cotsaris, E., Verhoeven, J. W., and Hush, N. S., 1987, J. Amer. chem. Soc., 109, 3258.

[23] Warman, J. M., de Haas, M. P., Paddon-Row, M. N., Cotsaris, E., Hush, N. S., Oevering, H., and Verhoeven, J. W., 1986, Nature, 320, 615.

[24] Bowler, B. E., Raphael, A. L., and Gray, H. B., 1990, Progress in Inorganic Chemistry: Bioiorganic Chemistry, Vol. 38, edited by S. J. Lippard (New York: John Wiley) p. 259.

[25] DeVault, D., 1984, Quantum-mechanical Tunnelling in Biological Systems (Cambridge: Cambridge University Press).

[26] Rice, S. A., 1985, Comprehensive Chemical Kinetics, Vol. 25, edited by C. H. Bamford, C. F. H. Tipper, and R. G. Compton (Amsterdam: Elsevier).

[27] Song, L., Dorfman, R. C., Swallen, S. F, and Fayer, M. D., 1991, J. phys. Chem., 95, 3454.

[28] Eads, D. D., Periasamy, N., and Fleming, G. R., 1989, J. chem. Phys., 90, 3876.

[29] Murata, S., and Tachiya, M., 1996, J. Chim. Phys., 93, 1577.

[30] Eads, D. D., Dismer, B. G., and Fleming, G. R., 1990, J. Chem. Phys., 93, 1136.

[31] Dutt, G. B., and Periasamy, N., 1991, J. chem. Soc. Faraday Trans., 87, 3815.

[32] Venkatamaran, B., Periasamy, N., Modi, S., Dutt, G. B., and Doraiswamy, S., 1992, Spectrochimica Acta, 48, 1707.

[33] Murata, S., and Tachiya, M., 1996, J. phys. Chem., 100, 4064.

[34] Pilling, M. J., and Rice, S. A., 1975, J. chem. Soc. Farsaday Trans., ii, 71, 1563.

[35] Szaвo, A., 1989, J. phys. Chem., 93, 6929.

[36] Burshtein, A. I., and Frantsuzov, P. A., 1992, J. Luminescence, $\mathbf{5 1 ,} 215$.

[37] Dorfman, R. C., Tachiya, M., and Fayer, M. D., 1991, Chem. Phys. Lett., 179, 152.

[38] Dorfman, R. C., and Fayer, M. D., 1992, J. chem. Phys., 96, 7410.

[39] Burshtein, A. I., 1992, Chem. Phys. Lett., 194, 247.

[40] Burshtein, A. I., Krissinel, E. B., and Mikhelashvili, M. S., 1994, J. phys. Chem., 98, 7319.

[41] Burshtein, A. I., and Shokhirev, N. V., 1997, J. Phys. Chem. A, 101, 25.

[42] Burshtein, A. I., and Frantsuzov, P. A., 1997, J. chem. Phys., 106, 3948.

[43] Steiner, U. E., and Ulrich, Th., 1989, Chem. Rev., 89, 51.

[44] Mclauchlan, K. A., and Steiner, U. E., 1991, Molec. Phys., 73, 241.

[45] Pedersen, J. B., and Freed, J. H., 1972, J. chem. Phys., 57, 1004.
[46] Pedersen, J. B., and Freed, J. H., 1973, J. chem. Phys., 58, 2746.

[47] Pedersen, J. B., and Freed, J. H., 1973, J. chem. Phys., 59, 2869.

[48] Pedersen, J. B., and Freed, J. H., 1974, J. chem. Phys., 61, 1517.

[49] Adrian, F. J., 1970, J. chem. Phys., 53, 3374.

[50] Adrian, F. J., 1971, J. chem. Phys., 54, 3912.

[51] Kanter de, F. J. J., Hollander den, J. A., Huizer, A. H., and Kaptein, R., 1977, Molec. Phys., 34, 857.

[52] Bittl, R., and Schulten, K., 1990, Chem. Phys. Lett., 173, 387.

[53] Bittl, R., and Schulten, K., 1989, J. chem. Phys., 90, 1794.

[54] Staerk, H., Busman, H.-G., Kühnle, W., and Treichel, R., 1991, J. phys. Chem., 95, 1906.

[55] Busmann, H.-G., Staerk, H., and Weller, A., 1989, J. chem. Phys., 91, 4098.

[56] Werner, U., and Staerk, H., 1995, J. phys. Chem., 99, 248.

[57] Steiner, U. E., and Bürssner, D., 1990, Z. phys. Chem. NF, 196, 159.

[58] Bürssner, D., Wolff, H.-J., and Steiner, U. E., 1993, Z. phys. Chem. NF, 182, 297.

[59] Bürssner, D., Wolff, H.-J., and Steiner, U. E., 1994, Angew. Chem. Intl. Edn., 33, 1772.

[60] Gilch, P., Linsenmann, M., Haas, W., and Steiner, U. E., 1996, Chem. Phys. Lett., 254, 384.

[61] Gilch, P., Pöllinger-Dammer, F., Musewald, C., Steiner, U. E., and Michel-Beyerle, M. E., 1998, Science, 281, 982.

[62] Banci, L., Bertini, I., and Luchinat, C., 1991, Nuclear and Electron Relaxation: The Magnetic Nucleus-Unpaired Electron Coupling in Solution (Weinheim: Verlag Chemie).

[63] Closs, G. L., and Doubleday, C. E., 1973, J. Amer. chem. Soc., 95, 2735.

[64] Weller, A., Staerk, H., and Treichel, R., 1984, J. chem. Soc. Faraday Discuss., 78, 271, 332.

[65] Staerk, H., Treichel, R., and Weller, A., 1986, Biophysical Effects of Steady Fields, edited by G. Maret, J. Kiepenheuer, and N. Boccara (Berlin: Springer-Verlag) p. 85 .

[66] Weller, A., 1987, Supramolecular Photochemistry, edited by V. Balzani (Dordrecht: Reidel) p. 343.

[67] Wolff, H.-J., Bürssner, D., and Steiner, U. E., 1995, Pure appl. Chem., 67, 167.

[68] Green, N. J. B., Pilling, M. J., and Clifford, P., 1989, Molec. Phys., 67, 1085.

[69] Burshtein, A. I., and Krissinel, E., 1998, J. phys. Chem. A, 102, 816.

[70] Burshtein, A. I., and Sivachenko, A. Yu., 1999, Chem. Phys., in press.

[71] Krissinel, E. B., and Shokhirev, N. V., 1989, Finitedifference Approximation of Spin and Anisotropic Problems of Diffusion Kinetics, Preprint of the Institute of Chemical Kinetics and Combustion, Siberian Branch of the USSR Academy of Sciences, Novosibirsk, USSR.

[72] Krissinel, E. B., Shokhirev, N. V., and Salikhov, K. M., 1989, Chem. Phys., 137, 207.

[73] Mints, R. G., and Pukhov, A. A., 1984, Chem. Phys., 87, 467.

[74] Salikhov, K. M., Molin, Yu. N., Sagdeev, R. Z., and Buchachenko, A. L., 1984, Spin polarization and 
Magnetic Effects in Radical Reactions (Amsterdam: Elsevier).

[75] Burshtein, A. I., Zharikov, A. A., Shokhirev, N. V., Krissinel, E. B., and Spirina, O. B., 1991, J. chem. Phys., 95, 8013.

[76] Lüders, K., and Salikhov, K. M., 1987, Chem. Phys., $117,113$.
[77] Maple V, Version 5 (University of Waterloo, Austin: Waterloo Maple Software).

[78] Abell, M., and Braselton, J., 1994, The Maple V Handbook (London: Academic Press).

[79] Dodrell, D. M., Pegg, D. T., Bendall, M. R., and Gregson, A. K., 1977, Aust. J. Chem., 30, 1635. 